\title{
Cardiac stem cell trials and the new world of cellular reprogramming: Time to move on
}

\author{
Todd K. Rosengart, MD, FACS, ${ }^{a}$ Vivek Patel, MD, ${ }^{a}$ and Frank W. Sellke, MD $^{\mathrm{b}}$
}

\footnotetext{
From the ${ }^{\mathrm{a}}$ Michael E. DeBakey Department of Surgery, Baylor College of Medicine, Houston, Tex; and ${ }^{\mathrm{b}}$ Division of Cardiothoracic Surgery, Brown Medical School, Providence, RI.

Received for publication Sept 15, 2017; revisions received Nov 9, 2017; accepted for publication Nov 16, 2017; available ahead of print Jan 31, 2018

Address for reprints: Todd K. Rosengart, MD, FACS, Michael E. DeBakey Department of Surgery, 6501 Fannin St, Suite NC114, Houston, TX 77030 (E-mail: todd.rosengart@bcm.edu).

J Thorac Cardiovasc Surg 2018;155:1642-6

0022-5223/\$36.00

Copyright (c) 2017 by The American Association for Thoracic Surgery

https://doi.org/10.1016/j.jtcvs.2017.11.104
}

Feature Editor's Note-Over the past 2 decades, researchers have achieved significant milestones in bringing stem cell regenerative medicine from bench to beside for the treatment of cardiovascular diseases. Despite these achievements and an evolving understanding of the potential mechanism of benefit from cardiogenesis to angiogenesis, cell recruitment, paracrine or immunomodulation effect, only very modest gains in clinical benefit have been documented from numerous translational and clinical studies. Significant challenges have been encountered, including appropriate cell type, dosing and frequency of administration, and cell retention and viability among several other important issues. Although the hype and hope of administration of exogenous stem cells to replace or recruit myocardium is quieting, important alternative discoveries and strategies have arisen that again bring exciting possibilities to the field.

In this issue of the Journal, Rosengart and colleagues have provided us an intriguing look into the new cardiac regeneration strategy based on several different and important discoveries, including the observations that somatic cells can be programed into induced pluripotent stem cells and that differentiated cells can be dedifferentiated or transdifferentiated into other types of differentiated cells. As discussed by Rosengart and colleagues, these discoveries open up tremendous possibilities and alternative approaches to cardiac repair, including the reprograming of fibroblast into cardiomyocytes or conversion of myofibroblasts back into nonfibrogenic states rather than using previous strategies of exogenous stem cell administration. Certainly, there are a number of important issues with cell reprogramming strategies, including the efficiency in the rates of transdifferentiation of cells that represent important hurdles. However, the article by Rosengart and colleagues is timely and important in that it challenges those in the field to pursue unconventional strategies offered by the cell therapy. in this field.

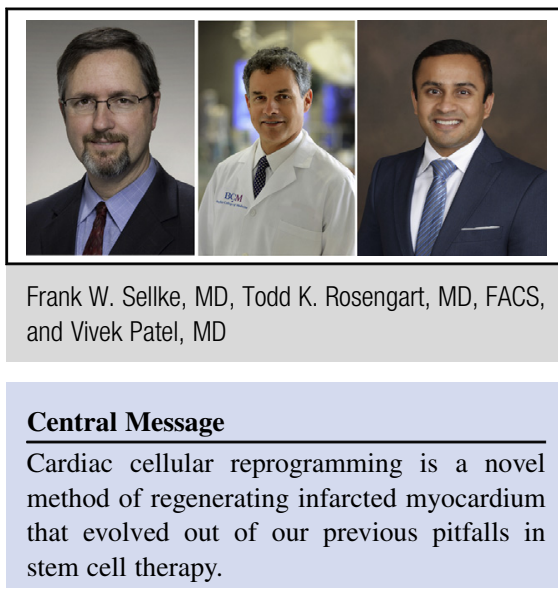

See Editorial Commentary page 1647.

techniques of cellular reprograming and wisely calls upon us to incorporate past lessons from the earlier era of stem

\section{Francis D. Pagani, MD}

The ever-quickening pace of new discoveries benefited by advances in our understanding of human genomics and other methodologies seems to be making yesterday's breakthroughs seem naive and antiquated at lightning speed. This may be the case for cardiac stem cell therapies, which less than 2 decades ago were heralded as a breakthrough technology for building new heart muscle and rescuing infarcted myocardium after myocardial infarction. Despite great promise in innumerable animal studies, the delivery of exogenous stem cells has, however, for a variety of reasons, been largely unsuccessful in human trials, and it seems it may be well past time to turn the page on original thinking

Just as the door of cardiac stem cell therapy appears to be closing, newly arrived is a new cardiac regeneration strategy, headlined by the intriguing premise of cardiac cellular reprogramming - a biologic intervention that allows the in situ conversion of fibroblasts into "induced cardiomyocytes," thereby replacing scar with regenerated myocardium. ${ }^{1}$ This exciting, next-generation approach to cardiac regeneration unthinkable only a few years ago was ushered in by the Nobel Prize-winning work of Yamanaka and colleagues, ${ }^{2}$ who reported in 2006 their discovery of a pathway to convert adult somatic cells into induced pluripotent stem 
(iPS) cells. Advanced by Srivastava and colleagues only 4 years after Yamanaka and colleague's seminal insights into cellular dedifferentiation, the revolutionary concept of direct cardiac cellular reprograming showed that not only could differentiated cells be dedifferentiated, they could also be transdifferentiated (without passing through a stem cell stage) into another type of differentiated cell; specifically, from fibroblast to cardiomyocyte. ${ }^{1}$

In broader terms, although the myocardium has long been considered a largely terminally differentiated tissue, it is ever more rapidly being recognized as a highly complex tissue, with a variety of ongoing cellular transdifferentiating processes occurring as the heart adapts to ischemia and other stresses. Although dogma had long held that any hope of myocardial regenerative therapy would require the delivery of exogenous progenitor cells to the heart because it was devoid of such cells endogenously, a growing body of evidence suggests that the myocardium may well be responsive to biologic manipulations that can stimulate endogenous myocardial regeneration. ${ }^{3}$ As but one example of the rapid expansion of this new knowledge, in the context of the not-too-distant discovery of cardiac progenitor cells (CPCs) as a potential in situ source of cardiomyocytes after cardiac injury, a recent article by Yaniz-Galende and colleagues ${ }^{4}$ has now identified subpopulations of CPCs that may (possibly deleteriously) preferentially differentiate into fibroblasts that will promote myocardial fibrosis.

In the context of this new knowledge, in addition to "classic" antifibrotic (eg, anti-transforming growth factor- $\beta$ ) strategies, a growing array of strategies are being devised to attack congestive heart failure on a variety of fronts, including strategies to (1) induce cardiomyocyte replication; (2) inhibit cellular differentiation pathways (eg, endothelial-mesenchymal transition) that promote the development of activated, fibrogenic fibroblasts, termed myofibroblasts; (3) convert myofibroblasts back into nonfibrogenic or otherwise-inactive states (eg, mesenchymalendothelial transition); (4) stimulate CPC expansion and differentiation; (5) activate myocardial telocytes-a newly discovered, specialized cell that is thought to support cardiomyocyte renewal, enhance angiogenesis, and decrease cardiac fibrosis; and (6) reprogram fibroblasts into (induced) cardiomyocytes (iCMs). ${ }^{3}$ Taken together, these strategies reflect a new understanding that a bifurcated fate awaits cardiac cells: under fibrogenic signaling, they may become myofibroblasts and promote pathologic fibrotic myocardial scaring; aided by external interventions, they can be redirected to a cardiomyocyte differentiation, and myocardial regeneration. ${ }^{3}$ The new focus in the field of myocardial regeneration is to shift the balance of this fibrosis:myoregeneration axis toward the latter and thereby promote endogenous myocardial recovery.

Given this exciting array of new biologic approaches to treating congestive heart failure, it is important to carefully consider the current status of cardiac stem cell therapy and the road taken by the field in getting here. Most importantly, it will be critical to ensure that any lessons learned are carried forward into these new therapeutic endeavors. To be exact, "stem cell therapy" refers to the strategy of administering exogenous stem cells or reserve cells (eg, embryonic stem cells, mesenchymal stem cells [MSCs], iPS cells, CPC cells, or skeletal myoblasts), targeting zones of myocardial infarction to regenerate functional myocardial tissue in these regions of postinfarct scarring and thereby at least partially normalize cardiac function. ${ }^{3}$ Despite the early promise of stem cell delivery in animal models, very few of the great number of stem cell clinical trials, encompassing thousands of patients and including more than 40 randomized controlled trials, have yielded compelling evidence of clinical benefit in improving heart function.

These disappointing outcomes have likely arisen for a variety of initially unrecognized challenges. These include (1) difficulties in retaining exogenous injected cells in the myocardium, especially in a distribution that will allow them to align and functionally integrate with host tissues; (2) extremely poor $(<1 \%-2 \%)$ implantation efficiency and survival of potentially immunogenic cells in the hostile milieu of the ischemic scar environment; and (3) inadequate availability of appropriate stem cell implant phenotypes and/ or differentiation into functional cardiomyocytes. ${ }^{6-9}$ Although strategies such as angiogenic pretreatment or cotreatment of hostile, infarcted implant territories, or incorporation of stem cells in a variety of biologic scaffolds have been explored to improve cell engraftment and survival as a solution to some of these challenges, none of these approaches has transitioned into clinical strategies. ${ }^{3}$

Of the variety of cell types tested as candidate cell transplants, the largest experience exists for bone marrow-derived hematopoietic (mononuclear) progenitor cells, which were the first true progenitor cell to be implanted, with more than 100 phase I to II trials of this cell type that included thousands of subjects. Despite the promising safety profile of these cell implants and results of trials, such as the STARheart (Stem Cell Transplantation in 191 Patients with Chronic Heart Failure) study showing improvements in ejection fraction (EF) of approximately $10 \%$, most other trials failed to demonstrate efficacy or have found clinically insignificant marginal improvements. ${ }^{6,10,11}$

Similar results can be seen in trials of other stem cell candidates. The SEED (Safety and Efficacy of Intracoronary Adult Human Mesenchymal Stem Cells After Acute Myocardial Infarction) randomized controlled trial in which 30 subjects received MSCs yielded a 6\% improvement in EF, but the PRECISE (Adipose-derived Regenerative Cells in Patients with Ischemic Cardiomyopathy) randomized controlled trial yielded no difference in EF for the 21 patients receiving MSCs versus placebo controls. ${ }^{10}$ Similarly, the controversial SCIPIO (Cardiac Stem 
Cells in Patients with Ischemic Cardiomyopathy) phase I trial of CPCs yielded about a $10 \%$ improvement in EF, but the CADUCEUS (Cardiosphere-Derived Cells for Heart Regeneration After Myocardial Infarction) trial did not yield improved EF (although a 12\% decrease in infarct size was reported in this relatively small trial of 17 treated patients). ${ }^{10}$

Three of the most recently published stem cell studies report similarly disappointing outcomes. The IMPACTCABG (Implantation of Autologous CD133+ Stem Cells in Patients Undergoing Coronary Artery Bypass Grafting) trial using autologous $\mathrm{CD} 133^{+}$endothelial progenitor cells injected intramyocardially as an adjunct to coronary artery bypass grafting yielded no difference in improvement in $\mathrm{EF}^{12}$; the RENEW (Reduction Coil Treatment in Patients With Emphysema) trial using autologous $\mathrm{CD} 34^{+}$cells injected intramyocardially demonstrated an improvement in angina frequency (relative risk $0.63, P=.05$ ) but no difference between groups in exercise tolerance testing and was terminated prematurely ${ }^{13}$; and the ALLSTAR (Allogeneic Heart Stem Cells to Achieve Myocardial Regeneration) phase II CPC trial-the sequel to the CADACEUS phase I CPC trial-also failed to demonstrate the same results. ${ }^{10}$ What is intriguing is that there often is a small benefit observed in these trials. This suggests that a better understanding of cell signaling and other advances may make cell therapy a viable treatment option in the (distant?) future.

What conclusions that can be drawn from the many stem cell trials now reported in the literature is perhaps best found in the growing number of meta-analyses and systematic review of this literature. In this regard, in one such review of 20 trials involving nearly 800 patients with chronic heart failure, the average improvement in EF was found to be less than $5 \%$, although stem cell therapy was associated with a lower mortality at 12 months (relative risk 0.42). ${ }^{11}$ Likewise, a recent Cochrane analysis of cell therapy in patients with acute heart failure after myocardial infarction (2732 patients, 41 randomized-controlled trials) demonstrated an average improvement in EF of less than $5 \% .^{5}$ The authors of this meta-analysis noted, however, that even this level of significant difference in outcome was eliminated once studies without appropriate blinding were excluded from their analysis.

In this context, new hope for the stem cell field was recently heralded with Yamanaka and colleagues' discovery of iPS cells, but investigators have again considered implantation of these cells without resolving the existing challenges of exogenous cell delivery: cell retention and long-term survival, electromechanical integration into the ischemic myocardium, and suitable phenotype and functionality. Not surprisingly, variable improvement in cardiac function has been seen with iPS cell implantation in small animal models, and added challenges of iPS immunogenicity and of teratoma formation have been newly identified in at least 3 studies. ${ }^{14}$

In the context of these findings, most investigators have largely abandoned the premise that stem cell implantation leads to differentiation of these cells into nascent cardiomyocytes contributing primary contractile or physiomechanical benefits to scarred myocardium. Instead, observed benefits of exogenous stem cell therapy have now alternatively been ascribed to putative angiogenic or other paracrine effects of these cells on host cardiomyocytes and other cells, which might support ischemic tissue neovascularization, resident myocyte survival, recruitment of endogenous CPCs, or cardiac remodeling. ${ }^{7,9,15}$ Certainly, these pathways may explain the sporadic success of stem cell trials, which do occasionally result in improvement in EF and reduction in fibrosis. Nevertheless, our understanding of such mechanisms remains elementary at this stage.

Given the lack of reproducible results over decades and the absence of a clearly defined mechanism of action of stem cells on cardiac regeneration, it would appear that new approaches are needed if myocardial regeneration is to become a reality. In this context, many of the challenges of stem cell therapy would appear to be resolved by the attributes of cellular reprogramming, wherein the abundant resource of fibroblasts generated at sites of postinfarct ventricular remodeling could be targeted for in situ conversion ("transdifferentiation") directly into iCMs. Specifically, a direct cellular reprogramming strategy, if sufficiently efficient, would theoretically enable the abundant regeneration of myocardial tissue from scar, and because it is an "in situ" strategy (ie, it targets endogenous cells, rather than requiring the injection of exogenous cells into the myocardium), it would largely abrogate the current challenges of exogenous stem cell expansion, injection, electrophysiological integration, and survival in the host myocardial syncytium. ${ }^{1,6,16}$ Of course, although appealing theoretically, it must first be cautioned that cellular reprograming, although novel, may in the end fail to provide any added benefit over the injection of exogenous stem cells. Many of the negative influences that have prevented other strategies from working may remain even with cellular reprograming.

With the caveat of wishing to avoid irrational enthusiasm, it can be reported that direct cellular reprograming studies have already demonstrated dramatic improvements in small animal myocardial infarction models, with $30 \%$ to $40 \%$ increases in postinfarct $\mathrm{EF}$ and up to $50 \%$ improvements in fibrosis observed in a growing number of studies. ${ }^{1,16}$ Underlying this favorable case for myocardial regeneration may be the apparent "default" tendency of cells to differentiate toward a cardiomyocyte phenotype, recapitulating the primacy of cardiac development in embryogenesis, which can be facilitated by the "therapeutic" delivery of various combinations of 
transcription factor master gene switches that are associated with early embryonic cardiac development.

Especially with this promising start, it will be critically important as this new science unfolds for investigators and observers of this field to not repeat the mistakes of the past 2 decades in investigations of stem cell interventions. To date, investigations in this field have thus far remained in the small animal arena. It is encouraging that these efforts have resulted in a comprehensive understanding of this new molecular biology to optimize its potential and resolve its challenges before moving into large animal, preclinical models or clinical trials before these questions are answered. This course will hopefully allow this field to avoid the pitfalls that grew to encumber the development of stem cells as a clinically applicable therapy.

To this end, while the first successful direct cardiac reprogramming, as reported by Srivastava and colleagues, achieved reprogramming efficiencies of about $7 \%$ using administration of the cardiodifferentiating transcription factors Gata4, Mef2c, and Tbx5, it is encouraging that investigators almost immediately after the report of Srivastava and colleagues began to better decipher the genetic pathways leading to cardiac cellular differentiation and (successfully) test alternative reprogramming cocktails to enhance reprogramming efficiency. ${ }^{1,16}$ In addition to the incorporation of alternative reprogramming factors in reprogramming cocktails, elucidation of better stoichiometry of transcription factor ratios, use of alternative gene transfer vectors and polycistronic vectors to improve cell targeting, and introduction of small molecule alternates to transcription factor genes quickly followed over the past 5 years since Srivastava and colleagues' landmark foray in this field. ${ }^{16,17}$

"Erasing" the somatic cell signature of target fibroblasts by downregulating native fibroblast gene expression has arisen as an intriguing and still newer approach to enhance cellular reprogramming efficiency. Small molecule inhibitors of key profibrotic pathways, targeting such profibrotic mediators as transforming growth factor- $\beta$ or Rhoassociated kinase, have also been thus used to enhance reprogramming efficiency and quality, as evidenced by the percentage of contractile cells and maturation of sarcomeric structures found in cultures of transdifferentiated cells. ${ }^{16}$ This and related strategies now often incorporate multitargeted microRNAs to improve the efficiency of reprogramming cocktail components. ${ }^{16}$

Encouragingly, as studies have moved from in vitro assays to in vivo modeling, it also appears that the myocardial microenvironment is more permissive to the formation of mature transdifferentiated cardiomyocytes than is that provided in cell culture, and reprogramming efficacy and improvements in cardiac function seen in vivo have largely exceeded expectations based on in vitro data. ${ }^{1,16,18}$ In this regard, it has been suggested that the in vivo milieu may include unknown paracrine signaling pathways as well as electrical and mechanical stimuli likely to promote the maturation of iCMs. ${ }^{16,18}$ In the context of these encouraging in vivo data, adjuvant prevascularization of the ischemic, scarred myocardium has been shown to further enhance $\mathrm{iCM}$ population density and improvements in postinfarct ventricular function after cell reprogramming. ${ }^{19}$

In contrast, it has also recently been uncovered that human cells, as opposed to the rodent cells in which most work in the field was being performed, are disproportionately resistant to reprogramming. ${ }^{16}$ One plausible explanation for this resistance relates to the tighter epigenetic controls on gene activation existent in higher-order species. $^{20}$ Investigators have already launched a new strategy to address this challenge, incorporating silencing of epigenetic barriers to lower the threshold in human cells to reprogramming stimuli. These efforts have included downregulation of the antiplasticity gene p63, cousin of p53. ${ }^{21}$ Interestingly, downregulation of p63, which has a redundant role in the cell, has not lead to uncontrolled proliferation or tumorigenic properties in in vitro studies but must of course be investigated in vivo.

Although the initial results of cellular reprogramming in small animals are promising, these data should also be interpreted with caution, as much more rigorous investigation into safety and efficacy of reprogramming factors, delivery methods, and long-term effects must still be established. It may therefore be prudent to perform preclinical studies in more clinically relevant large animal models before the initiation of clinical trials using cellular reprograming. As these efforts unfold, to avoid the missteps of previous cardiac biologic interventions, it will no doubt be important to further test reprogramming efficacy under conditions such as hypercholesterolemia and diabetes, which have been shown to alter cell signaling and lessen the effectiveness of regenerative strategies. ${ }^{22,23}$ In addition, collaboration from other disciplines investigating novel delivery techniques and the replacement of reprogramming factors with pharmacotherapies will perhaps make cardiac cellular reprogramming a much more feasible clinical therapy.

The possible success of such biologic interventions for the treatment of congestive heart failure remain as important as ever, because even the most advanced of current therapies, including transplantation or ventricular assist devices, are associated with considerable morbidity; limiting cost and/or availability; and aggregate suboptimal 5 -year mortality rates of less than $50 \% .{ }^{24}$ So, the wisdom that has hopefully been garnered from the false starts and unproven premises of the stem cell therapy field over the past 2 decades will also hopefully provide a solid starting point for well-designed studies using soundly selected therapeutic agents and routes of delivery to test the potential clinical efficacy of new myocardial regeneration strategies, 
including direct cellular reprogramming. Efforts to pursue these best options will be critical if this new field avoids the pitfalls of stem cell therapy, which has enrolled thousands of study candidates that been subjected to risks-in many cases, perhaps needlessly and fruitlessly.

\section{Conflict of Interest Statement}

Authors have nothing to disclose with regard to commercial support.

The work represented from our lab, The Laboratory for Cardiac Regeneration at Baylor College of Medicine, was funded by the National Heart Lung and Blood Institute (R01 HL121294-01A1).

\section{References}

1. Qian L, Huang Y, Spencer CI, Foley A, Vedantham V, Liu L, et al. In vivo reprogramming of murine cardiac fibroblasts into induced cardiomyocytes. Nature. 2012;485:593-8.

2. Takahashi K, Yamanaka S. Induction of pluripotent stem cells from mouse embryonic and adult fibroblast cultures by defined factors. Cell. 2006;126:663-76.

3. Cahill TJ, Choudhury RP, Riley PR. Heart regeneration and repair after myocardial infarction: translational opportunities for novel therapeutics. Nat Rev Drug Discov. 2017;16:699-717.

4. Yaniz-Galende E, Roux M, Nadaud S, Mougenot N, Bouvet M, Claude O, et al Fibrogenic potential of PW1/Peg3 expressing cardiac stem cells. J Am Coll Cardiol. 2017;70:728-41.

5. Fisher SA, Zhang H, Doree C, Mathur A, Martin-Rendon E. Stem cell treatment for acute myocardial infarction. Cochrane Database Syst Rev 2015;9:CD006536.

6. Rosengart TK, Fallon E, Crystal RG. Cardiac biointerventions: whatever happened to stem cell and gene therapy? Innovations (Phila). 2012;7:173-9.

7. Segers VF, Lee RT. Stem-cell therapy for cardiac disease. Nature. 2008;451:937-42.

8. Lalit PA, Hei DJ, Raval AN, Kamp TJ. Induced pluripotent stem cells for postmyocardial infarction repair: remarkable opportunities and challenges. Circ Res. 2014:114:1328-45.

9. Batty JA, Lima JA, Kunadian V. Direct cellular reprogramming for cardiac repair and regeneration. Eur J Heart Fail. 2015;18:145-56.

10. Hao M, Wang R, Wang W. Cell therapies in cardiomyopathy: current status of clinical trials. Anal Cell Pathol (Amst). 2017;2017:9404057.

11. Fisher SA, Doree C, Mathur M, Martin-Rendon E. Meta-analysis of cell therapy trials for patients with heart failure. Circ Res. 2015;116:1361-77.
12. Forcillo J, Stevens LM, Mansour S, Prieto I, Salem R, Baron C, et al. Implantation of CD133 + stem cells in patients undergoing coronary bypass surgery: IMPACT-CABG pilot trial. Can J Cardiol. 2013;29:441-7.

13. Povsic TJ, Henry TD, Traverse JH, Fortuin FD, Schaer GL, Kereiakes DJ, et al. The RENEW trial: efficacy and safety of intramyocardial autologous CD34(+) cell administration in patients with refractory angina. JACC Cardiovasc Interv. 2016;9:1576-85.

14. Lang CI, Wolfien M, Langenbach A, Muller P, Wolkenhauer O, Yavari A. Cardiac cell therapies for the treatment of acute myocardial infarction: a meta-analysis from mouse studies. Cell Physiol Biochem. 2017;42: 254-68.

15. Xu J, Lian W, Li L, Huang Z. Generation of induced cardiac progenitor cells via somatic reprogramming. Oncotarget. 2017;8:29442-57.

16. Patel V, Mathison M, Singh VP, Yang J, Rosengart TK. Direct cardiac cellular reprogramming for cardiac regeneration. Curr Treat Options Cardiovasc Med. 2016;18:58.

17. Mathison M, Singh VP, Gersch RP, Ramirez MO, Cooney A, Kaminsky SM, et al. "Triplet" polycistronic vectors encoding Gata4, Mef2c, and Tbx5 enhances postinfarct ventricular functional improvement compared with singlet vectors. J Thorac Cardiovasc Surg. 2014;148:1656-64.e2.

18. Song K, Nam YJ, Luo X, Qi X, Tan W, Huang GN, et al. Heart repair by reprogramming non-myocytes with cardiac transcription factors. Nature. 2012;485: 599-604.

19. Mathison M, Gersch RP, Nasser A, Lilo S, Korman M, Fourman M, et al. In vivo cardiac cellular reprogramming efficacy is enhanced by angiogenic preconditioning of the infarcted myocardium with vascular endothelial growth factor. $J \mathrm{Am}$ Heart Assoc. 2012;1:e005652.

20. Ebrahimi B. Reprogramming barriers and enhancers: strategies to enhance the efficiency and kinetics of induced pluripotency. Cell Regen (Lond). 2015;4:10.

21. Patel V, Cooney A, Flores ER, Singh VP, Mathison M, Sanagasetti D, et al. Unlocking reprogramming capability: silencing antiplasticity gene p63 enhances the reprogramming of fibroblasts into induced cardiomyocytes. Circ Res. 2016; 119:A33.

22. Ruel M, Wu GF, Khan TA, Bianchi C, Voixine P, Li JY, et al. Inhibited angiogenic response to surgical FGF-2 protein therapy in a swine model of endothelial dysfunction. Circulation. 2003;108(suppl II):335-40.

23. Sodha NR, Clements RT, Boodhwani M, Xu S-H, Laham RJ, Bianchi C, et al. Endostatin and angiostatin are increased in diabetic patients with coronary artery disease and associated with impaired coronary collateral formation. Am J Physiol Heart Circ Physiol. 2009;296:H428-34.

24. Mozaffarian D, Benjamin EJ, Go AS, Arnett DK, Blaha MJ, Cushman M, et al. Heart Disease and Stroke Statistics 2016 Update: a report from the American Heart Association. Circulation. 2016;133:e38-360. 